\title{
Physical vs. Virtual Agent Embodiment and Effects on Social Interaction
}

\author{
Sam Thellman, Annika Silvervarg, Agneta Gulz and Tom Ziemke \\ Conference Publication
}

\section{Tweet}

N.B.: When citing this work, cite the original article.

Original Publication:

Sam Thellman, Annika Silvervarg, Agneta Gulz and Tom Ziemke, Physical vs. Virtual Agent Embodiment and Effects on Social Interaction, Intelligent Virtual Agents, 2016. (), pp.412415.

http://dx.doi.org/10.1007/978-3-319-47665-0_44

Copyright: www.springer.com

Postprint available at: Linköping University Electronic Press

http://urn.kb.se/resolve?urn=urn:nbn:se:liu:diva-132940

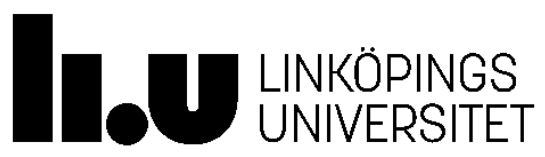




\title{
Physical vs. virtual agent embodiment and effects on social interaction
}

\author{
Sam Thellman, Annika Silvervarg, Agneta Gulz \& Tom Ziemke ${ }^{凶}$ \\ Dept. of Computer and Information Science, Linköping University, Linköping, Sweden \\ \{sam.thellman, annika.silvervarg, agneta.gulz, \\ tom.ziemke\}@liu.se
}

\begin{abstract}
Previous work indicates that physical robots elicit more favorable social responses than virtual agents. These effects have been attributed to the physical embodiment. However, a recent meta-analysis by Li [1] suggests that the benefits of robots are due to physical presence rather than physical embodiment. To further explore the importance of presence we conducted a pilot study investigating the relationship between physical and social presence. The results suggest that social presence of an artificial agent is important for interaction with people, and that the extent to which it is perceived as socially present might be unaffected by whether it is physically or virtually present.
\end{abstract}

Keywords: Embodiment, physical presence, social presence, social influence, social robots, virtual agents.

\section{Introduction}

Experimental work comparing social robots with virtual agents has shown that robots typically elicit more favorable social responses than virtual agents. A recently published meta-analysis by Li [1], based on 62 statistically significant differences between physical and virtual agents observed in 33 experimental works, found that in $73 \%$ of cases physical robots were found to achieve more positive results, in $21 \%$ virtual agents worked better, and $6 \%$ of the results involved crossover interaction effects that varied depending on participant age, task type, and presence of robot gestures. Hence, the overall outcome of Li's meta-analysis is that robots in general-or on average-are more effective than virtual agents in social interaction contexts. Furthermore, $\mathrm{Li}$ concluded that the benefits of robots are due to their physical presence, i.e. their co-location with users, rather than their physical embodiment/bodies.

To further explore the importance of physical presence to social interaction we investigated the relationship between physical and social presence. There is evidence that social presence, defined by Lee [2, p. 41] as "the experience of artificial objects as social actors that manifest humanness", is an important factor in social interaction (e.g. [3, 4]). The relationship between physical and social presence is highly relevant to the design of artificial agents, in particular to the choice of whether to implement a robotic or virtual agent for the purpose of social interaction. We therefore conducted a 
pilot study based on the conjecture that (H1) a physically present agent will elicit a higher level of perceived social presence compared to a virtually present agent.

Moreover, we investigated whether Li's finding regarding the benefits of physically over virtually present agents held true in the case of two specific agents - a physical robot and a virtual robot. Since it is not possible to measure all qualities associated with satisfactory social interaction skills in a single study, we approached the question of what characterizes successful social interaction with a focus on the importance of social influence to successful interaction. Based on Li's findings, and the notion that successful social interaction skills are characterized by the ability to influence the behavior of others, we conjectured that (H2) a physically present agent will have higher social influence than a virtually present agent.

Finally, we tested the hypothesis that (H3) an artificial agent which is perceived as highly socially present will have a higher social influence, regardless of its physical or virtual presence. The purpose of testing this hypothesis was to determine whether social presence is an important factor to successful social interaction in the context of the specific types of interactions that took place in the study.

\section{Our Study}

Participants (an opportunity sample of 60 university students, mostly computer science; 40 males, 20 females; median age 23) were randomly assigned to interact with either a physical or a virtual Nao robot (Figure 1) in an in-between group experiment (i.e. every subject only encountered one type of agent).
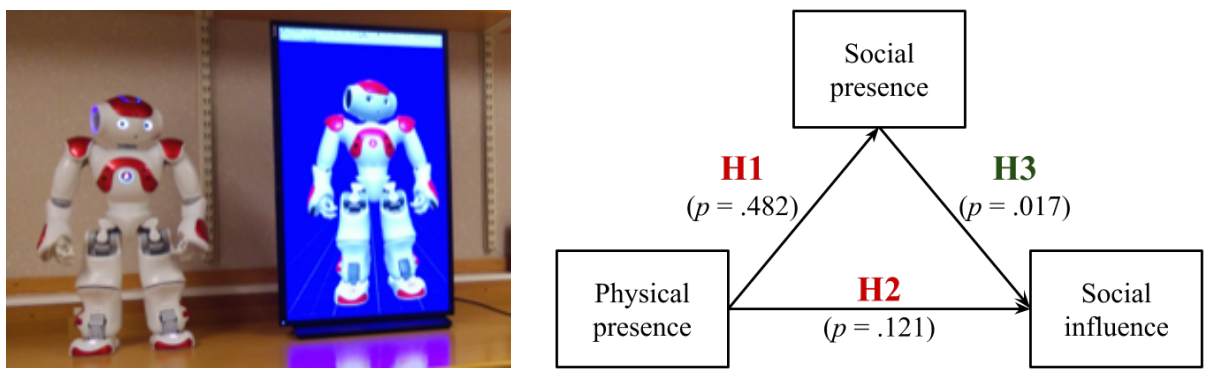

Figure 1. Left: physical Nao robot, produced by Aldebaran Robotics, and its virtual counterpart. Right: summary of statistical results (supporting H3, but not $\mathrm{H} 1$ and H2; details below).

We employed the ultimatum game (UG) experimental paradigm to assess the effect of physical presence (i.e., physical versus virtual robot) on the social presence (H1) and social influence (H2) of the agent, as well as the effect of the social presence of the agent on its social influence (H3). UG is used to study bargaining behavior in the field of experimental economics [5] and has also been used in human-robot interaction studies (e.g. [6]). It can be used to measure social influence based on the assumption that the extent to which others are willing to accept an offer made by an individual is a reliable indicator of the degree of social influence of that individual, relative to the social influence of other individuals making the same offer. We used a one-shot 
variant of the UG where the human participant responded to a single offer proposed by the robot. The rules of the game were as follows. A sum of money-in our case the amount of 100 SEK (apx. \$12) - was to be distributed between two players. It was up to the first player, "the proposer" (in this case the robot), to suggest how to divide the sum between the proposer and the second player, "the responder" (in this case the human). It was up to the responder to accept or reject the offer. If the responder accepted the offer, both would be compensated in accordance with the offer. If the responder declined, neither would receive any money.

\subsection{Procedure and Instruments}

Prior to the experiment each participant was informed about the procedure, treatment of experimental data, their rights as participants, and that they could receive economic compensation depending on actions taken in the experiment. Participants gave written consent. They were then instructed to sit facing the robot, and the interaction session was started. After explaining the rules to the participant, the robot gave an offer of 20 SEK (apx. \$2.3) and instructed the participant to press the green or the red button on a device placed before them to accept or reject the offer. After the session, participants were asked to fill out a questionnaire measuring social presence, adopted from [7] (translated to Swedish); the scale consisted of eight questions and had a high level of internal consistency, as determined by a Chronbach's $\alpha$ of .895. Finally, participants that had accepted the offer were compensated.

\section{$3 \quad$ Results}

A Mann-Whitney U test (suitable for ordinal numerical data) was run to assess the hypothesis that a physically present agent will elicit a higher level of perceived social presence than a virtually present agent $(\mathrm{H} 1)$. Median social presence scores for physical vs. virtual conditions (mean rank $=28.9$ vs. 32.1 ) were not found to be statistically different $(U=498, z=.703, p=.482)$. This result did not support $\mathrm{H} 1$.

A chi-square test for association was conducted between agent condition and participants' decision to accept or reject the robot's offer to assess the hypothesis that a physically present agent will be more socially influential than a virtually present agent (H2). Participant responses between agent conditions (13 vs. 19 accepts out of 30 possible for physical and virtual conditions respectively) were not statistically different $\chi^{2}(1)=2.41, p=.121$. This result did not support $\mathrm{H} 2$.

A second Mann-Whitney U test was run to assess H3. The median social presence score was statistically significantly higher for participants accepting the offer (6.69) than for those rejecting it (5.94), $U=610, z=2.395, p=.017$. This result supported H3: participants experiencing the robot as a highly social actor were more inclined to accept the offer, suggesting that social presence is important to social interaction.

The results are summarized in Figure 1 (right): physical versus virtual presence had no statistically significant effect on either social presence (H1) or social influence $(\mathrm{H} 2)$, but social presence had a statistically significant effect on social influence $(\mathrm{H} 3)$. 


\section{Discussion and Conclusion}

The result from testing $\mathrm{H} 3$ indicates that the social presence of an artificial agent is significant to its ability to successfully interact with others, at least in the context of the types of interaction featured in this study. From testing H1 and H2, we saw, contrary to our expectations, that our physically present agent was not perceived as more socially present than its virtually present counterpart, nor did it give rise to more favorable social responses. This suggests that physical presence and social presence might be independent from each other, i.e. the extent to which an agent is experienced as a "social actor that manifest humanness" [2, p. 41] might be unaffected by the choice of physical or virtual embodiment.

It should be noted that the pilot study presented here featured a domain-specific and relatively short participant task (one-shot UG), and robot behavior which included little speech and autonomy and no gestures. Whether the same effects will arise in other interaction scenarios is a topic for future research. Moreover, according to $\mathrm{Li}$ [1], comparisons between co-present robots and virtual agents - such as this studyrisk conflating two possibly distinct aspects of physical and virtual embodiment: the physicality of their embodiment, defined by Li as "the physical or digital state of an agent independent of how it is displayed to a user" and the physicality of their presence, i.e. "being either 'physically displayed' ... [or] 'digitally displayed"” [1, p. 25]. $\mathrm{Li}$ argues that the relevant comparison for the purposes of investigating effects of physical presence is that between a co- and tele-present (i.e., physically embodied but virtually present) robot. Future research should explore the relationship between physical and social presence and whether similar effects arise in other interaction scenarios, specifically in comparisons between co- and tele-present robots.

\section{$5 \quad$ References}

1. Li, J.: The Benefit of Being Physically Present: A Survey of Experimental Works Comparing Copresent robots, Telepresent Robots and Virtual Agents. Int. J. Hum-Comp. St. 77, 23-37 (2015)

2. Lee, K. M.: Presence, Explicated. Communication Theory 14(1), 27-50 (2004)

3. Pereira, A., Martinho, C., Leite, I., \& Paiva, A.: iCat, the Chess Player: the Influence of Embodiment in the Enjoyment of a Game, 7th Int. Conf. on Autonomous. Agents and Multiagent Syst., IFAAMAS, 1253-1256 (2008)

4. Kennedy, J., Baxter, P. \& Belpaeme, T.: Comparing Robot Embodiments in a Guided Discovery Learning Interaction with Children. Int. J. Soc. Rob. 7(2), 293-308 (2015)

5. Güth, W., Schmittberger, R., \& Schwarze, B.: An Experimental Analysis of Ultimatum Bargaining. J. Econ. Beh. Org. 3(4), 367-388 (1982)

6. Nitsch, V., \& Glassen, T. (2015). Investigating the Effects of Robot Behavior and Attitude toward Technology on Social Human-Robot Interactions. IEEE RO-MAN, 535-540 (2015)

7. Lee, K. M., Jung, Y., Kim, J., \& Kim, S. R.: Are Physically Embodied Social Agents Better than Disembodied Social Agents?: The Effects of Physical Embodiment, Tactile Interaction, and People's Loneliness in Human-Robot Interaction. Int. J. of Hum-Comp. St. 64(10), 962-973 (2006) 